\title{
Partisan biases in social information use
}

\author{
Lucas Molleman ${ }^{1 *}$, Andrea Gradassi ${ }^{1}$, Mubashir Sultan ${ }^{2,3}$, Wouter van den Bos ${ }^{1,3}$ \\ ${ }^{1}$ Psychology, University of Amsterdam, Amsterdam, The Netherlands \\ ${ }^{2}$ Institute for Interdisciplinary Studies, University of Amsterdam, Amsterdam, The Netherlands \\ ${ }^{3}$ Center for Adaptive Rationality, Max Planck Institute for Human Development, Berlin, Germany \\ * Corresponding author: I.s.molleman@uva.nl
}

Date: 21 May 2021

\begin{abstract}
Preferential learning from like-minded others can help individuals acquire adaptive knowledge and socially appropriate behaviour, but it can also reinforce echo chambers and fuel polarization. Ingroup bias is well-documented in the social transmission of opinions, attitudes, and values. However, important questions about its role in the integration of social information when forming factual beliefs are outstanding. We present a naturalistic yet controlled experiment showing that social information is most impactful when provided by ingroup rather than outgroup sources. Participants predicted the 2020 US elections by state and could adjust their predictions after observing the prediction of a Democrat or a Republican. Adjustments were largest when observing fellow-partisans, and when social information favoured the participants' party. Exploratory analyses reveal that these partisan biases are driven by Republican participants. Our findings help understand the variation of social information use along political orientations and its consequences for belief polarization in increasingly fragmented populations.
\end{abstract}




\section{Introduction}

Social information can guide behaviour when individuals are uncertain about how to solve a problem, help the formation of accurate beliefs, and supports the accumulation of adaptive knowledge in populations [1-3]. However, the transmission of social information can also have undesirable effects, including the spread of false news and the emergence of discord between different groups in society. The deep polarization evident in the United States (US) is a topical example, where supporters of the two major political parties persistently disagree about values and policies, such as the desirability of same-sex marriage or gun ownership. These disagreements can extend beyond such value-based matters and into basic facts [4,5], leading people from different groups in society to experience different realities, removing the foundations of debates and complicating consensus-building on important issues like election outcomes, safety of COVID-19 vaccines, and the speed of climate change [6-8].

Evidence suggests that the aforementioned issues stem largely from people's preferences for interacting with like-minded others, structuring their social networks in a way that disproportionately exposes them to self-concordant information [9]. In the US, social ties tend to form along partisan lines [10], producing strong segregation and filter bubbles, both offline [11] and online (e.g., on social media; [12-14]). However, above and beyond this biased exposure, individuals' responses to social information can also depend on the identity of its source. Indeed, ample empirical evidence has confirmed that people prioritize ingroup information over outgroup information when forming political beliefs and opinions [15-21]. For matters of fact and basic knowledge, people may also place more trust in the views of ingroup members, because they likely encounter similar problems [22]. In contrast, the views of outgroup members may be perceived as uninformed, biased, or ideologically motivated [5,23-25]. Little is known, however, about how ingroup biases in social information use operate in such factual domains, in which integrating the views of others can promote the formation of accurate beliefs about the world.

In this paper, we investigate how people integrate social information from partisan ingroup and outgroup members in belief formation. We present a pre-registered experiment in which US participants, who identified as either Democrats or Republicans, predicted the outcome of the 2020 US election by state and were paid for accuracy. Participants could adjust their predictions after observing the predictions of previous participants. We experimentally controlled the party affiliation of these social sources (to measure ingroup bias) and whether their predictions were more or less favourable to the participant's party (to measure motivated reasoning). The task represents a naturalistic and clearcut setting involving decisions under uncertainty in which participants could observe others to reduce decision uncertainty. We find that participants adjust their predictions more when social information is provided by fellow-partisans rather than opposing-partisans, and when it indicates a favourable outcome for their own party. Further exploratory analyses show that these biases are particularly pronounced among Republicans. Our study provides experimental evidence for ingroup biases in individuals' social information use, and highlights the importance of psychological processes in the emergence and persistence of between-group disagreement about basic facts. 


\section{Results}

Experimental Setup. One week prior to the 2020 US General Elections, we recruited 221 US participants (116 Democrats and 105 Republicans; see Table S1 for sample details). After indicating their party affiliation, participants predicted the election outcomes for 20 US states. For each state, they started by predicting what percentage of votes would go to either of the two major parties (Fig. 1A), and stated their confidence in this first prediction (low or high). Participants then observed the prediction of a participant who had previously completed the task, and could make a second prediction (Fig. 1B). We calculate social information use $(s)$ as relative adjustments from first to second predictions per state (see Fig. 1 for formal definition).

Our experiment controls the availability, the source, and direction of social information, without using deception. To measure ingroup bias in social information use, we presented participants with predictions from fellow-partisans in one experimental block and from opposing-partisans in another block. To test the potential effects of motivated reasoning, we varied the direction of social information within blocks. That is, relative to the participant's first prediction, the social information indicated sometimes more and sometimes less votes for the participant's favoured party. Both accuracy of predictions and confidence ratings were incentivised (see Methods for details; see https://osf.io/vksjy/ for preregistration).

A

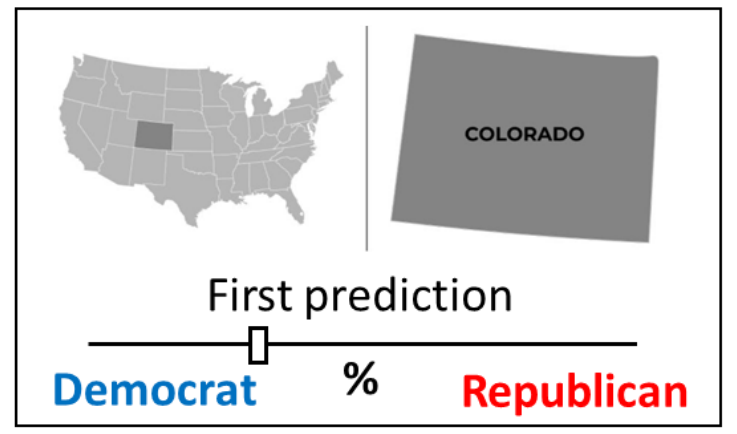

B

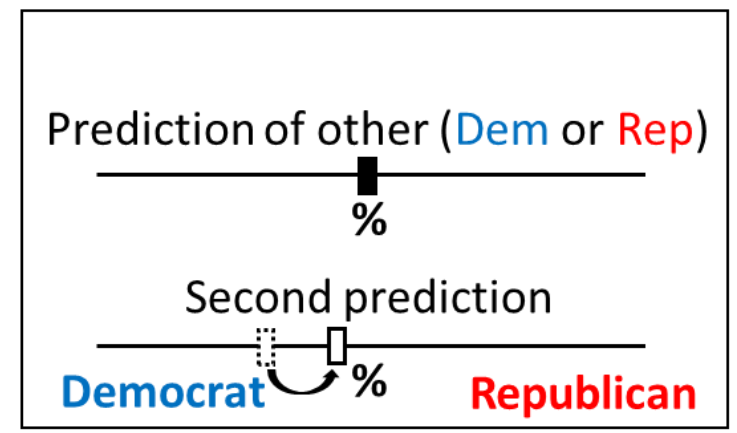

Fig. 1. Election prediction task. (A). For a series of US states, participants had to predict the distribution of 100 votes across the two major parties in the 2020 US General Elections. (B). After making this first prediction $\left(E_{1}\right)$, they observed the estimate of another participant $(X)$ and entered a second prediction $\left(E_{2}\right)$. Participants were awarded for accuracy (Materials and Methods). We experimentally varied the other participant's partisanship and the direction of social information (see Experimental Setup). Social information use in a round $(s)$ was calculated as the relative adjustment towards the other's prediction: $s=\left(E_{2}-E_{1}\right) /\left(X-E_{1}\right)$. The value of $s$ thus reflects the relative weight assigned to social information in forming the second prediction ( $s=0$ : ignoring social information $s=1$ : copying social information; $s=0.5$ weighing the own first prediction and social information equally).

Basic results. Partisanship in our sample was strong, with participants from both parties strongly identifying with their own party (Fig. S1). For most states, we observe a small but significant 'optimistic bias': On average, first predictions of Democrats were 4.2 percentage points more in favour of Democrats than were first predictions of Republicans (Fig. S2). Participants' confidence in their first 
predictions was positively correlated with the population size of the respective state, indicating that the task led to variation in uncertainty (Fig. S3).

In individual rounds, adjustments from first to second predictions were overwhelmingly in the range $0 \leq$ $s \leq 1$ (88\%; Fig. S4, S5). This implies that participants' second predictions $\left(E_{2}\right)$ were typically weighted averages of personal information $\left(E_{1}\right)$ and social information $(X)$. As pre-registered, the subsequent analyses focus on these cases only.

People prioritize personal over social information. Overall, participants adjusted $29 \%$ towards social information. This indicates that on average, participants assigned more than twice as much weight to their own first predictions than to social information, reflecting the widely documented phenomenon of 'egocentric discounting' [26,27].

People prioritize ingroup over outgroup social sources. Figure $2 \mathrm{~A}$ shows that social information had more impact when it was provided by a fellow-partisan (ingroup) than by an opposing-partisan (outgroup). The pre-registered statistical model confirms that adjustments were largest when social information stemmed from an ingroup source (Fig. 2B; Table S2; effect of 'Ingroup Source': $p=0.005$ ). Social information use markedly varied between individuals and was strongly correlated across blocks showing ingroup and outgroup sources (Fig. S6), confirming previous observations that individuals' social information use tends to be consistent across contexts [28-31].

A

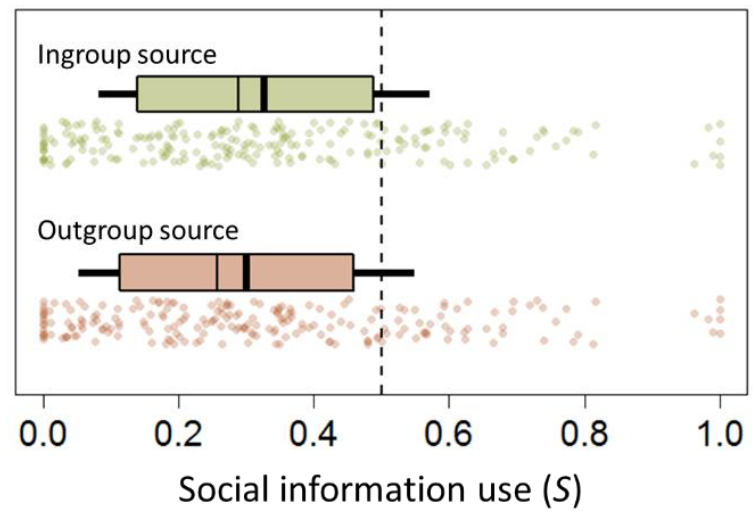

B

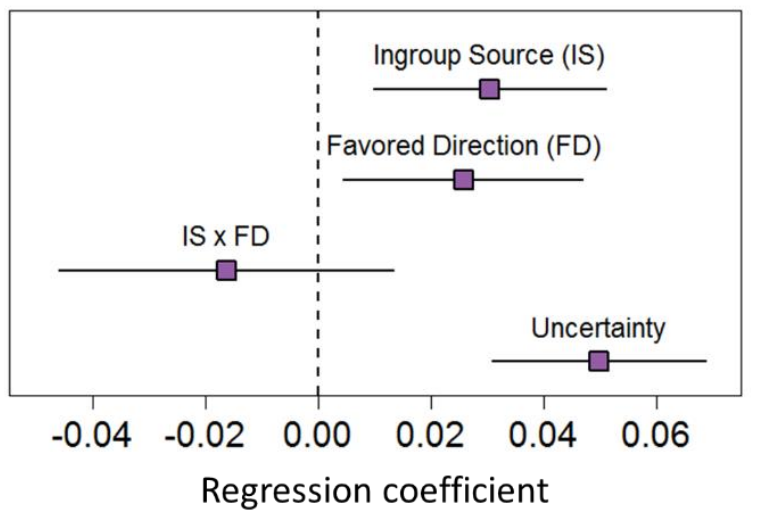

Fig 2. Social information use among partisans. A. Distributions of participants' mean adjustments $(S)$ when social information stemmed from a fellow-partisan or an opposing-partisan (box: first and third quartile; thin vertical line: median; thick vertical line and whiskers: mean and s.d.). The dotted vertical line indicates $S=0.5$, in which participants assign equal weight to their own first predictions and social information. Each participant is represented by a dot in each condition. B. Estimates of a linear mixed effects model fitted to adjustments in individual rounds, with bars showing $95 \% \mathrm{Cls}$ (for full numerical presentation, see Table S2). 'Ingroup Source' indicates whether the participant observed social information stemming from a fellow-partisan (1) or an opposing-partisan (0). 'Favoured Direction' indicates whether social information showed more (1) or less (0) votes for the participant's favoured party than their own first prediction. 'Uncertainty' indicates whether the participant rated low (1) or high (0) confidence in their own first prediction. 
Motivated reasoning. The model further shows that motivated reasoning impacts belief updating: adjustments were larger when social information showed a prediction in the direction favorable to the participant's affiliated party (Fig. 2B; Table S2; effect of 'Favored Direction': $p=0.019$ ). That is, participants adjusted their predictions more if social information showed a higher vote share for their preferred party than their own first prediction. This effect did not depend on the source of social information (the interaction effect 'Ingroup Source x Favored Direction' was not significant). Furthermore, participants adjusted significantly more when they were uncertain (vs certain) of their own first prediction.

Exploratory Analyses. Figure 3 shows that the observed ingroup biases were driven by participants who identified as Republican. We characterize party bias for each individual as the difference in mean adjustment when observing a participant from either party $\left(B=S_{\text {Republican }}-S_{\text {Democrat }}\right)$. We observe that the mean value of $B$ for Republican participants is significantly higher than zero (mean=0.033; one-sided ttest: $t=2.53$, d.f. $=100, p=0.006$ ), while for Democratic participants, it is not significantly lower than zero (mean=-0.009; $\mathrm{t}=-0.587$, d.f. $=114, p=0.279$; Fig. 3A). Participants reporting a stronger party affiliation tended to display a slightly stronger ingroup bias (Fig. S7).

A

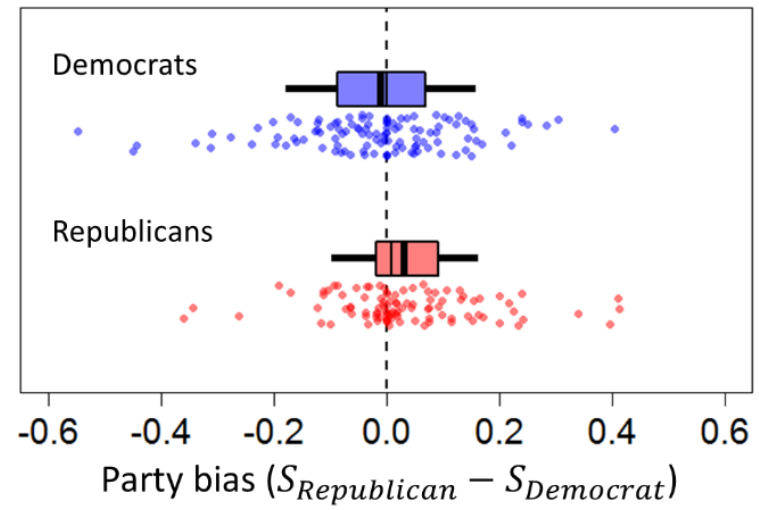

B

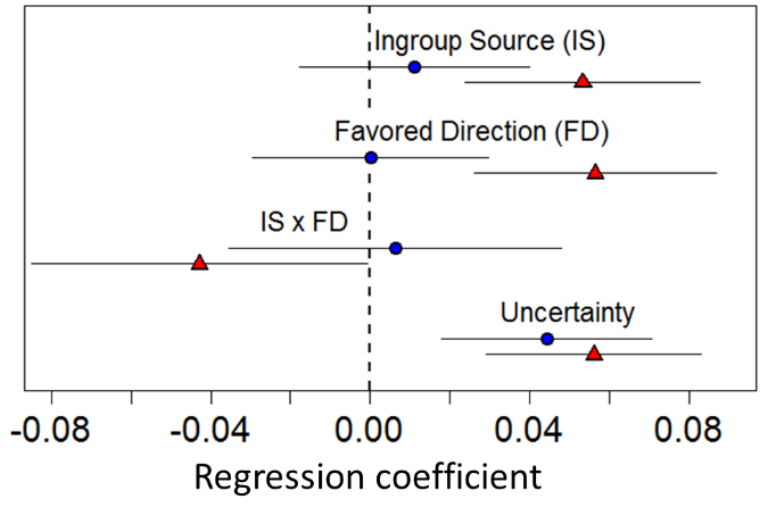

Fig. 3. Ingroup bias and motivated reasoning in social information use is driven by Republicans. (A) Partisan bias was driven by Republican participants. On the $x$-axis, positive and negative values indicate assigning more weight to social information from Republicans and Democrats, respectively. In both panels, each dot indicates one participant. (B) Estimates of a linear mixed effects model fitted to adjustments in individual rounds, using data from participants who identified as Democratic (blue circles) or Republican (red triangles), with bars showing $95 \% \mathrm{Cls}$ (for full numerical presentation, see Table S3). Labels as in Fig. 2B.

To further explore these results, we fitted the regression model reported in Fig. 2B to the adjustments from Democratic- and Republican-identifying participants separately. This analysis reveals that the partisan biases in social information use are driven by Republicans (Fig. 3B; Table S3). While adjustments of Democrats were not significantly impacted by the party identity of the social source, nor by motivated reasoning (95\% Cl's associated with the blue dots bracket zero), these effects were more pronounced in Republicans (red triangles). The negative interaction 'Ingroup Source x Favored Direction' 
( $p=0.048$; Table S3), however, suggests a moderating effect when Republicans observed a fellowpartisan predicting more favorable outcomes for their favored party.

\section{Discussion}

Our study provides empirical evidence of how partisanship impacts the integration of social information into people's beliefs. The results can be summarized in three main points. Firstly, our experiment reveals an ingroup bias in processing social information in the domain of facts: social information carries more weight when it stems from fellow-partisan rather than opposing-partisan social sources. Secondly, we report evidence of motivated reasoning: Social information impacts beliefs more when it signals better outcomes for one's own favored party. Finally, both ingroup bias and motivated reasoning in social information use are most pronounced among Republicans.

Participants on average incorporated social information more when it stemmed from fellow-partisans (Fig. 2A). This indicates that ingroup bias in social information use extends beyond the domains of values, attitudes, and opinions, and into matters of fact. These results cast doubt on the effectiveness of online attempts to mitigate polarization that expose people to a more balanced information diet by including information from opposing-partisan sources [32]. Rather, our results suggest that parallel realities are prone to persist when misinformation (or disinformation [33]) infects a group from within. Conversely, when people hold inaccurate beliefs (e.g., about vaccine safety or the speed of climate change), changing their mind is most probable when information stems from sources they identify with [34-36]. These insights may inform measures to promote the spread of accurate information within groups by enhancing the salience of group identity of knowledgeable individuals. Similarly, accurate information may more readily transmitted between groups when the salience of identities is reduced, e.g., when the name of opposing-partisan news sources are masked.

Social information had more impact when it indicated a preferred outcome for the participant's favored party (Fig. 2B). In our experiment, participants received monetary incentives for accuracy, and their decisions could not be observed by others, minimizing the scope for motivations to protect their (political) identity [25,37], preferences to affiliate with one's own group, or attempts to gain social status [5]. Nevertheless, in the anonymous and factual decision setting of the experiment, participants integrated information more when it signaled better outcomes for their favored party. Regardless of whether this result may reflect wishful thinking, or, conversely, selective discounting of displeasing information (i.e., deliberate ignorance; [38]), it underscores the pervasiveness of motivated reasoning in belief formation, beyond the domains of values, policies and opinions, into in the domain of facts.

Our exploratory analyses suggest that the observed effects of ingroup bias and motivated reasoning in social information use are driven by Republicans (Fig. 3). This is in line with research in political psychology showing that conservative-leaning individuals are particularly prone to heed evidence that reaffirms, rather than challenges their views $[20,39]$. Note, though, that within the groups of both Democratic- and Republican-identifying participants, there are substantial individual differences in ingroup bias and motivated reasoning. To understand the psychological mechanisms underlying these biases in social information use, it would be useful to examine their links with individual characteristics 
beyond party preference, including reflective thinking style $[25,40,41]$, intelligence $[42,43]$, and perceptions of outgroup hostility [44].

The results presented in this paper are based on behavior of participants from the US, in which Democrats and Republicans form relatively well-defined groups that share a long-standing antagonism. In political and non-political contexts, new group identities frequently arise with their specific demarcations and motives to distinguish themselves from other groups, or 'cancel' people that express discordant views $[15,34,45,46]$. Each of these identities can have its own influence on individuals' preferences about how the world should look like, and may shape their perceptions of the world $[5,24,47]$. In turn, this might increase the strength of ingroup biases and motivated reasoning in social information use and fracture social networks into more and more smaller groups. It remains an empirical question, however, whether and to what extent the biases in social information use observed in this study generalize to other (political or non-political) groups, both within US society or in different countries [48-50].

Our experiment allowed us to isolate the effects of ingroup biases and motivated reasoning in one-off updating events. This leaves open the important question of how these biases may compound and be amplified over the course of interactions to impact collective outcomes. In a social network, people repeatedly observe similar views when surrounded by like-minded others, who preferentially share information favourable to their own views (e.g., sharing online news items that cast their favored party in a positive light $[13,14,51])$, and repudiate discordant views. Together, these mechanisms may turn pre-existing between-group differences in beliefs (cf. Fig. S2) into divergent and entrenched positions, and render individuals' beliefs more inaccurate, risking substantial societal unrest when facing the facts (e.g., strong disappointment with-or even outright rejection of-election outcomes).

Our study adds to the literature aimed at understanding the emergence and persistence of partisan divides in society. The results highlight that biased exposure to social information from ingroups is not the only mechanism at the root of widespread disagreements: Individual cognition also plays an important role in the prioritization of information from ingroups over information from outgroups. Our results show that ingroup bias and motivated reasoning in integrating social information are not limited to values but also extend to the domain of facts. These mechanisms can limit the ability of groups within societies to agree on ground truths, making it impossible to reach consensus, and eventually threatening the foundations of the democratic process.

\section{Materials and Methods}

General procedures. Participants (mean age: 39 (s.d.=11); range 24-73; 46\% female) were invited to complete a task on Amazon Mechanical Turk (MTurk). Inclusion criteria required that participants be located in the US and have an approval rate of at least 95\%. The experiment was programmed using LIONESS Lab [52]. Ethical approval was given by the IRB of Psychology, University of Amsterdam (ref. 2020-DP-12605). We obtained informed consent before participants started the experiment. Participants on average took 20 minutes to complete the experiment. Mean earnings ranged from $\$ 1.70$ to $\$ 3.70$ (mean $\$ 2.56$ ), making for an hourly rate of $\$ 5.10-\$ 11.10$ (mean $\$ 7.68$ ). 
Election prediction task. The experiment was conducted in the week prior to the 2020 US General Elections. Participants started with a question about their party affiliation and another question about the degree of identification with their party. They then started the task in which they had to predict the election outcome of a series of 20 different US states. In each round, participants indicated how many out of 100 votes they expected to go to either the Democratic or the Republican party, and indicated their confidence in their prediction ('low' or 'high'). After entering this first prediction $\left(E_{1}\right)$, participants observed social information $(X)$ in the form of the prediction of another participant who completed this task before. Participants could enter a second prediction $\left(E_{2}\right.$; see Supplementary Text for screenshots of all experimental materials).

Quantity of interest. For each round we calculated a participant social information use $(s)$, as the adjustment from first prediction $\left(E_{1}\right)$ to second prediction $\left(E_{2}\right)$, as a fraction of the distance between their first estimate and social information $(X): s=\left(E_{2}-E_{1}\right) /\left(X-E_{1}\right)$. Rearranging this formula as $E_{2}=(1-s)$ $* E_{1}+s * X$ highlights that second predictions were an average of the first predictions and social information, weighted by $s$.

Experimental conditions. Prior to the main experiment, we pre-recorded individual predictions for each state from 100 MTurk workers. We rewarded them for accuracy (see below). We recorded their party affiliation (Democrat or Republican) and used their predictions as social information in the main experiment. Using this prerecorded pool of predictions allowed us to achieve experimental control without deception.

The main experiment consisted of two blocks (counterbalanced in order) of ten rounds each. In one block, the previous participant was a fellow-partisan, in another block, they were an opposing-partisan. Within blocks, we varied the direction of social information relative to the participant's own first prediction. In four rounds, social information showed a lower vote share for the participant's preferred party, and in another four rounds, social information showed a higher vote share. To allow for a constant scope for adjustments and to control for distance effects that might influence adjustments $[30,31,53]$, we held the difference between a participant's first prediction and social information constant at 15-18 percentage points. To enhance believability, the two remaining (filler) rounds, social information was either very close or very far from the participant's first prediction.

Incentives. Participants were rewarded for the accuracy of their predictions. After the election results were decided, we randomly selected a single first prediction, and another single second prediction for each participant to determine their bonus payment. Selection of the first prediction was weighted by participants' confidence, with a round with high confidence being twice as likely to be selected than a round with low confidence. Each second prediction was equally likely to be selected for payment. For each selected prediction, we paid $\$ 1.00$ if it exactly matched the election outcome for that state (rounded to the nearest percentile). For each percentage point off, we subtracted $\$ 0.05$. Participants in the prerecorded pool only made first predictions (no social information was shown to them), and hence, only these were used for selecting a random response for payment. Participants in the main experiment were informed that the participants whose predictions they could observe were also paid for accuracy (experimental materials are shown in full in the Supplementary Text). 
Data and code. Data and code is available via the publicly accessible repository: github.com/LucasMolleman/Partisanship

\section{References}

1. Richerson PJ, Boyd R. 2004 Not by genes alone: How culture transformed human evolution. University of Chicago Press.

2. Dean LG, Kendal RL, Schapiro SJ, Thierry B, Laland KN. 2012 Identification of the social and cognitive processes underlying human cumulative culture. Science 335, 1114-1118.

3. Henrich J. 2015 The Secret of Our Success: How Culture Is Driving Human Evolution, Domesticating Our Species, and Making us Smarter. Princeton University Press.

4. Robbett A, Matthews PH. 2018 Partisan bias and expressive voting. J. Public Econ. 157, 107-120. (doi:10.1016/j.jpubeco.2017.09.008)

5. Van Bavel JJ, Pereira A. 2018 The Partisan Brain: An Identity-Based Model of Political Belief. Trends Cogn. Sci. 22, 213-224. (doi:10.1016/j.tics.2018.01.004)

6. Jolley D, Douglas KM. 2014 The effects of anti-vaccine conspiracy theories on vaccination intentions. PloS One 9, e89177.

7. Lewandowsky S, Gignac GE, Vaughan S. 2013 The pivotal role of perceived scientific consensus in acceptance of science. Nat. Clim. Change 3, 399-404. (doi:10.1038/nclimate1720)

8. Murphy J et al. 2021 Psychological characteristics associated with COVID-19 vaccine hesitancy and resistance in Ireland and the United Kingdom. Nat. Commun. 12, 29. (doi:10.1038/s41467-02020226-9)

9. McPherson M, Smith-Lovin L, Cook JM. 2001 Birds of a Feather: Homophily in Social Networks. Annu. Rev. Sociol. 27, 415-444. (doi:10.1146/annurev.soc.27.1.415)

10. Mosleh M, Martel C, Eckles D, Rand DG. 2021 Shared partisanship dramatically increases social tie formation in a Twitter field experiment. Proc. Natl. Acad. Sci. 118. (doi:10.1073/pnas.2022761118)

11. Brown JR, Enos RD. 2021 The measurement of partisan sorting for 180 million voters. Nat. Hum. Behav. , 1-11.

12. Lazer DMJ et al. 2018 The science of fake news. Science 359, 1094-1096. (doi:10.1126/science.aao2998)

13. Garimella K, De Francisci Morales G, Gionis A, Mathioudakis M. 2018 Political discourse on social media: Echo chambers, gatekeepers, and the price of bipartisanship. In Proceedings of the 2018 World Wide Web Conference, pp. 913-922.

14. Cinelli M, Morales GDF, Galeazzi A, Quattrociocchi W, Starnini M. 2021 The echo chamber effect on social media. Proc. Natl. Acad. Sci. 118. (doi:10.1073/pnas.2023301118)

15. Baumeister RF, Leary MR. 1995 The need to belong: desire for interpersonal attachments as a fundamental human motivation. Psychol. Bull. 117, 497.

16. Bartels LM. 2000 Partisanship and voting behavior, 1952-1996. Am. J. Polit. Sci. , 35-50.

17. Cohen GL. 2003 Party over policy: The dominating impact of group influence on political beliefs. J. Pers. Soc. Psychol. 85, 808.

18. Gerber AS, Huber GA, Washington E. 2010 Party affiliation, partisanship, and political beliefs: A field experiment. Am. Polit. Sci. Rev. 104, 720-744.

19. Petersen MB, Skov M, Serritzlew S, Ramsøy T. 2013 Motivated reasoning and political parties: Evidence for increased processing in the face of party cues. Polit. Behav. 35, 831-854. 
20. Jost JT. 2017 Ideological Asymmetries and the Essence of Political Psychology. Polit. Psychol. 38, 167-208. (doi:https://doi.org/10.1111/pops.12407)

21. Ditto PH, Liu BS, Clark CJ, Wojcik SP, Chen EE, Grady RH, Celniker JB, Zinger JF. 2019 At least bias is bipartisan: A meta-analytic comparison of partisan bias in liberals and conservatives. Perspect. Psychol. Sci. 14, 273-291.

22. Tajfel H, Turner J. 2004 The social identity theory of intergroup behavior. Abridged version reprinted in J. Jost \& J. Sidanius. Polit. Psychol. Key Read. , 276-293.

23. Vallone RP, Ross L, Lepper MR. 1985 The hostile media phenomenon: biased perception and perceptions of media bias in coverage of the Beirut massacre. J. Pers. Soc. Psychol. 49, 577.

24. Ross L, Ward A. 1995 Naive realism in everyday life: Implications for social conflict and misunderstanding.

25. Kahan DM. 2012 Ideology, motivated reasoning, and cognitive reflection: An experimental study. Judgm. Decis. Mak. 8, 407-24.

26. Yaniv I, Kleinberger E. 2000 Advice Taking in Decision Making: Egocentric Discounting and Reputation Formation. Organ. Behav. Hum. Decis. Process. 83, 260-281.

(doi:10.1006/obhd.2000.2909)

27. Morin O, Jacquet PO, Vaesen K, Acerbi A. 2021 Social information use and social information waste. Philos. Trans. R. Soc. B Biol. Sci. 376, 20200052. (doi:10.1098/rstb.2020.0052)

28. Toelch U, Bruce MJ, Newson L, Richerson PJ, Reader SM. 2014 Individual consistency and flexibility in human social information use. Proc. R. Soc. B Biol. Sci. 281, 20132864.

29. Molleman L, van den Berg P, Weissing FJ. 2014 Consistent individual differences in human social learning strategies. Nat. Commun. 5, 3570. (doi:10.1038/ncomms4570)

30. Molleman L, Kurvers RH, van den Bos W. 2019 Unleashing the BEAST: a brief measure of human social information use. Evol. Hum. Behav. 40, 492-499.

31. Molleman L, Tump AN, Gradassi A, Herzog S, Jayles B, Kurvers RHJM, van den Bos W. 2020 Strategies for integrating disparate social information. Proc. R. Soc. B Biol. Sci. 287, 20202413. (doi:10.1098/rspb.2020.2413)

32. Bozdag E, van den Hoven J. 2015 Breaking the filter bubble: democracy and design. Ethics Inf. Technol. 17, 249-265.

33. Freelon D, Wells C. 2020 Disinformation as Political Communication. Polit. Commun. 37, 145-156. (doi:10.1080/10584609.2020.1723755)

34. Fukuyama F. 2018 Identity: The demand for dignity and the politics of resentment. Farrar, Straus and Giroux.

35. Guilbeault D, Becker J, Centola D. 2018 Social learning and partisan bias in the interpretation of climate trends. Proc. Natl. Acad. Sci. 115, 9714-9719.

36. Pink S, Chu J, Druckman J, Rand D, Willer R. 2021 Elite Party Cues Increase Vaccination Intentions among Republicans. (doi:10.31234/osf.io/f9jq5)

37. Kahan DM. 2017 Misconceptions, misinformation, and the logic of identity-protective cognition.

38. Hertwig R, Engel C. 2020 Deliberate ignorance: Choosing not to know. Strungmann Forum Reports.

39. Boutyline A, Willer R. 2017 The social structure of political echo chambers: Variation in ideological homophily in online networks. Polit. Psychol. 38, 551-569.

40. Deppe KD, Gonzalez FJ, Neiman JL, Jacobs C, Pahlke J, Smith KB, Hibbing JR. 2015 Reflective liberals and intuitive conservatives: A look at the Cognitive Reflection Test and ideology. Judgm. Decis. Mak. 10, 314-331. 
41. Pennycook G, Rand DG. 2019 Cognitive reflection and the 2016 US Presidential election. Pers. Soc. Psychol. Bull. 45, 224-239.

42. Hodson G, Busseri MA. 2012 Bright minds and dark attitudes: Lower cognitive ability predicts greater prejudice through right-wing ideology and low intergroup contact. Psychol. Sci. 23, 187-195.

43. Muthukrishna M, Morgan TJ, Henrich J. 2016 The when and who of social learning and conformist transmission. Evol. Hum. Behav. 37, 10-20.

44. Ruggeri $\mathrm{K}$ et al. 2021 The general fault in our fault lines. Nat. Hum. Behav. , 1-11.

45. Brewer MB. 1991 The social self: On being the same and different at the same time. Pers. Soc. Psychol. Bull. 17, 475-482.

46. Abrams D, Hogg MA. 1990 Social Identification, Self-Categorization and Social Influence. Eur. Rev. Soc. Psychol. 1, 195-228. (doi:10.1080/14792779108401862)

47. Turner JC, Oakes PJ, Haslam SA, McGarty C. 1994 Self and collective: Cognition and social context. Pers. Soc. Psychol. Bull. 20, 454-463.

48. Mesoudi A, Chang L, Dall SR, Thornton A. 2016 The evolution of individual and cultural variation in social learning. Trends Ecol. Evol. , 215-225.

49. Molleman L, Gächter S. 2018 Societal background influences social learning in cooperative decision making. Evol. Hum. Behav. 39, 547-555. (doi:10.1016/j.evolhumbehav.2018.05.007)

50. Van Leeuwen EJC, Cohen E, Collier-Baker E, Rapold CJ, Schäfer M, Schütte S, Haun DBM. 2018 The development of human social learning across seven societies. Nat. Commun. 9, 2076. (doi:10.1038/s41467-018-04468-2)

51. Del Vicario M, Bessi A, Zollo F, Petroni F, Scala A, Caldarelli G, Stanley HE, Quattrociocchi W. 2016 The spreading of misinformation online. Proc. Natl. Acad. Sci. 113, 554-559.

52. Giamattei M, Yahosseini KS, Gächter S, Molleman L. 2020 LIONESS Lab: a free web-based platform for conducting interactive experiments online. J. Econ. Sci. Assoc. (doi:10.1007/s40881-020-000870)

53. Jayles B, Kim H, Escobedo R, Cezera S, Blanchet A, Kameda T, Sire C, Theraulaz G. 2017 How social information can improve estimation accuracy in human groups. Proc. Natl. Acad. Sci. , 201703695.

Acknowledgments. We thank the members of the Connected Minds lab at the University of Amsterdam and the Max Planck Research Group Dynamics of Social Behavior for valuable feedback and discussions. 


\section{Supplementary Materials}

belonging to

\section{Partisan biases in social information use}

by Lucas Molleman*, Andrea Gradassi, Mubashir Sultan, and Wouter van den Bos

*Corresponding author: I.s.molleman@uva.nl

Contents:

Supplementary Figures S1 - S7

Supplementary Tables S1 - S3

Experimental materials 


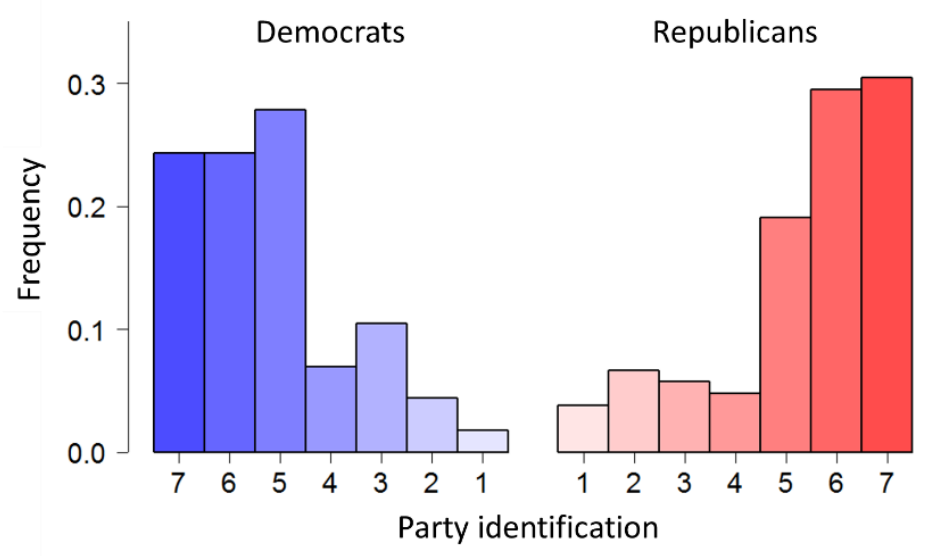

Fig. S1. Party identification in our sample. Prior to starting the experimental task, participants were asked to indicate their preferred party (Democrats or Republicans) and their degree of identification with that party (on a 7-point scale). Overall, partisanship was strong: mean identification for the 116 Democrats was 5.3 (s.d. =1.5) and for the 105 Republicans was 5.4 (s.d.=1.7). 


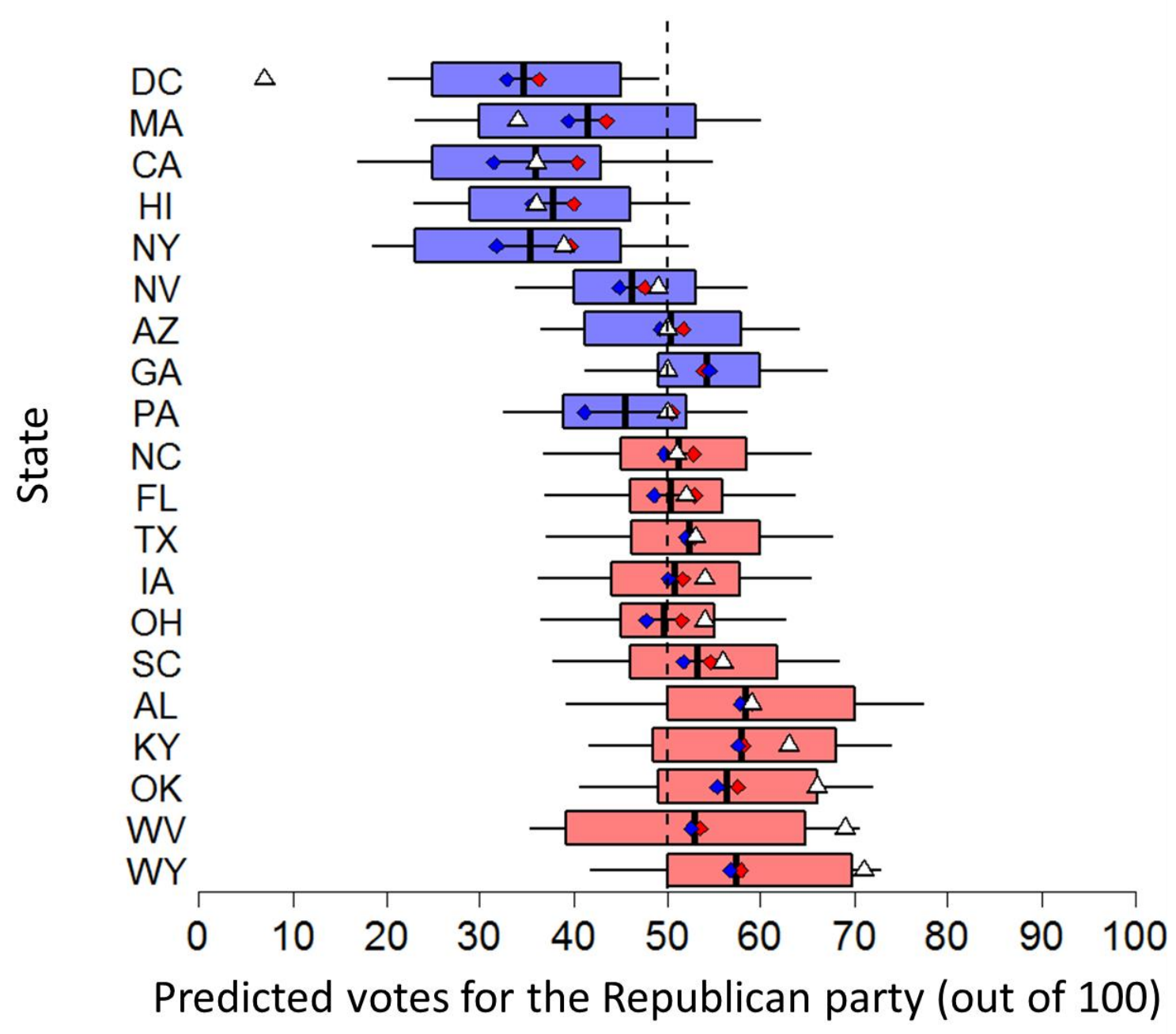

Fig. S2. First predictions by state. For each of the 20 states in our experiment we show the distribution of participants' first predictions (boxes: quartiles; vertical lines: means; whiskers: standard deviations). Blue and red diamonds (connected with horizontal lines) show mean first predictions of Democratic and Republican participants, respectively. White triangles indicate the actual outcomes of the General Election (which took place one week after the experiment; blue and red box backgrounds indicate victories for the Democratic and Republican party, respectively). We observe that for most states, the first and third quartiles of the distribution bracketed the outcome. This indicates that overall, participants were quite accurate in their predictions. Exceptions are the (relatively small) states of Washington D.C., Oklahoma, West Virginia and Wyoming. On average, Republicans predicted 4.2 more votes (out of 100) for Republicans than Democrats did. Similarly, participants from both parties were also optimistic about the chances of the candidate of their preferred party to win the Presidency (Democrats: mean=70\% (s.d.=23); Republicans (73\%; s.d.=28); predictions not incentivised). 


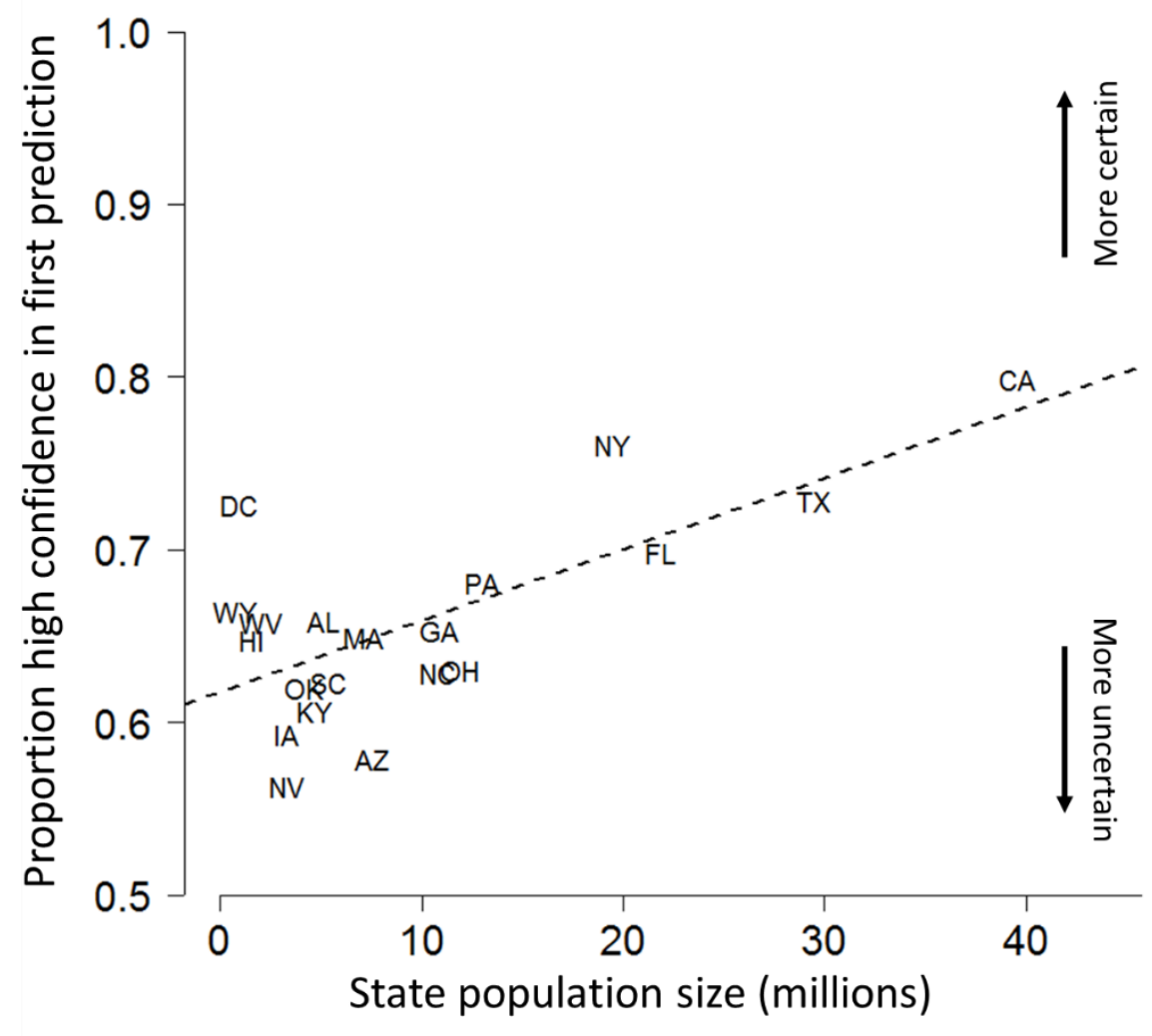

Fig. S3. Proportion of participants indicating high confidence in their own prediction as a function of the population size of each state. We observe a strong positive association (Pearson's $r=0.71$, d.f. $=18$, $p<0.001$ ), indicating that the larger the state, the more confident people were in their first predictions. First predictions associated with high confidence turned out to be on average 3.2 percentage points closer to the election outcome than those with low confidence (linear mixed model with 'participant' as random intercept fitted to log-transformed absolute deviations of participant's first predictions from the actual outcome: $t=3.98 ; p<0.001)$. 


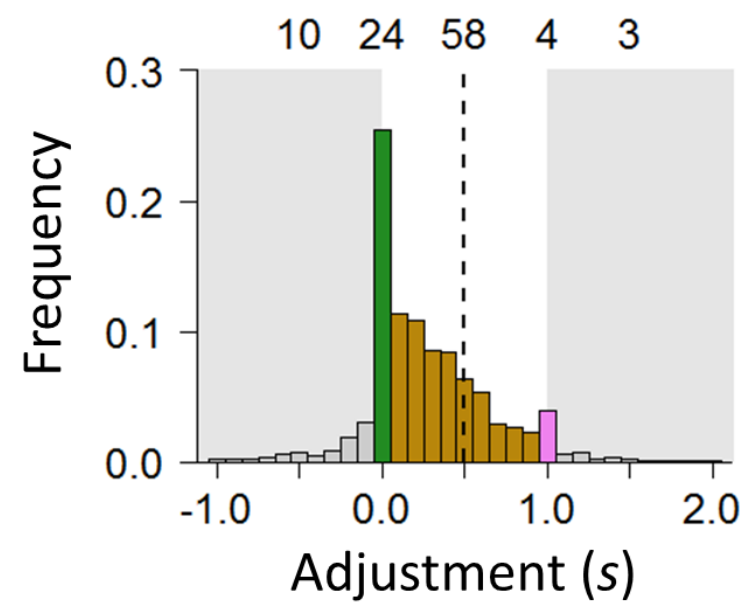

Fig. S4. Distribution of adjustments in individual rounds. Bars indicate frequencies of adjustments from first to second predictions after observing social information. The white background shows the expected range of $0 \leq s \leq 1$, in which participants' second predictions were a weighted average of their own first prediction and social information (see Molleman et al 2019 for details). Bar colors indicate qualitatively different cases of keeping the first prediction ( $24 \% s=0$; green), copying social information ( $4 \% s=1$; pink), and compromising between the two ( $58 \% 0<\mathrm{s}<1$; yellow). Grey bars and band backgrounds indicate cases where participants moved away from social information $(s<0)$, or adjusted beyond the social information $(s>1)$. We omit data from the 'filler' rounds in which social information was not controlled to be at an intermediate distance from participants' first estimates (see Methods for details). Numbers up top show the percentages for each of the different cases. For distributions broken down by party identify of the participant and the source of social information, see Fig. S5. 


\section{Party affiliation of the social source}
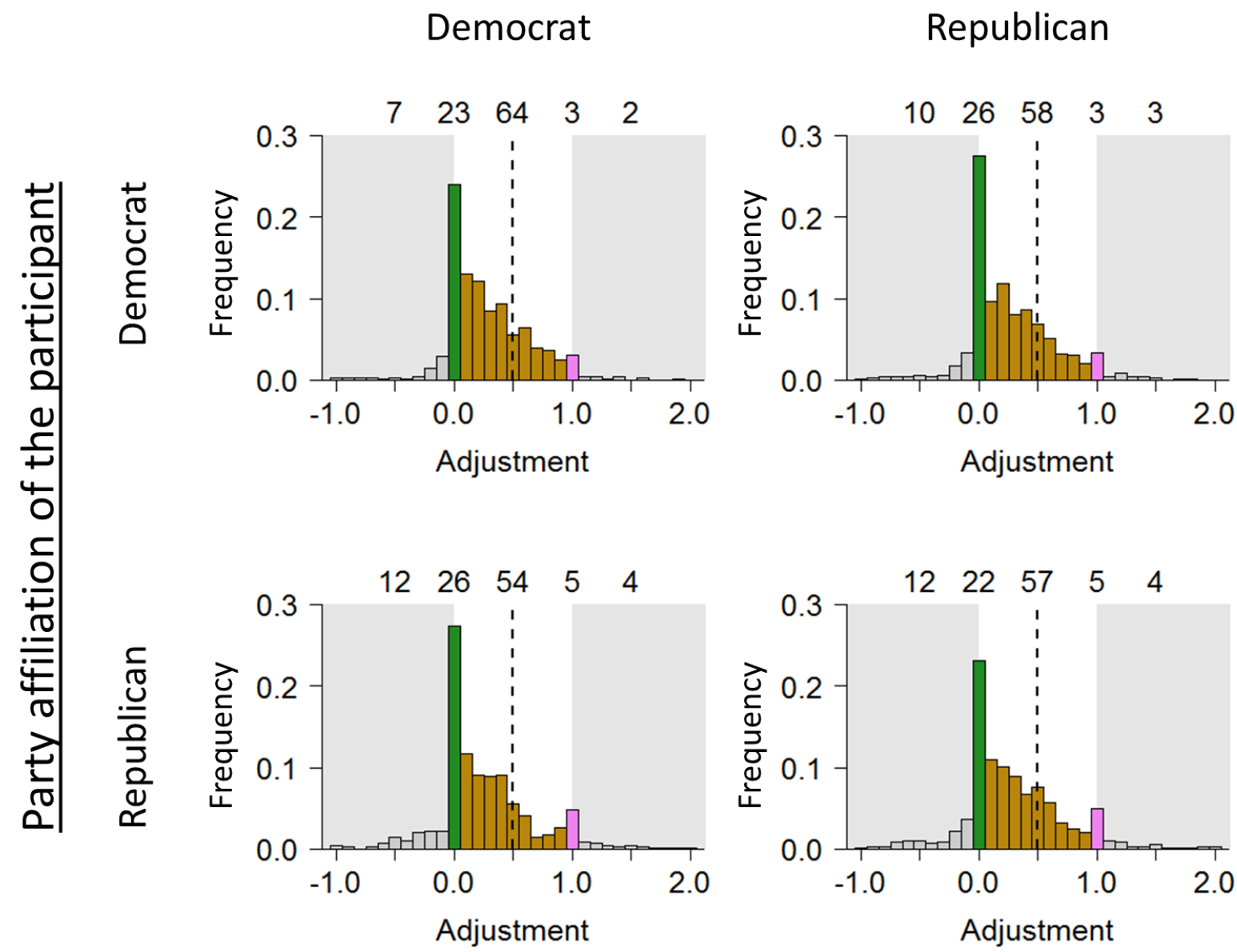

Fig. S5. Distribution of adjustments in individual rounds, broken down by party affiliation of the participant and the social source. Panels as in Fig. S4. The likelihood of moving away from social information $(s<0)$ did not significantly vary with the party affiliation of the participant nor its social source, or the interaction between the two (logistic generalized linear model with 'participant' as random intercept: $\mathrm{P}>0.066$ for main effects of 'own party', 'other's party' and the interaction between the two). 
A

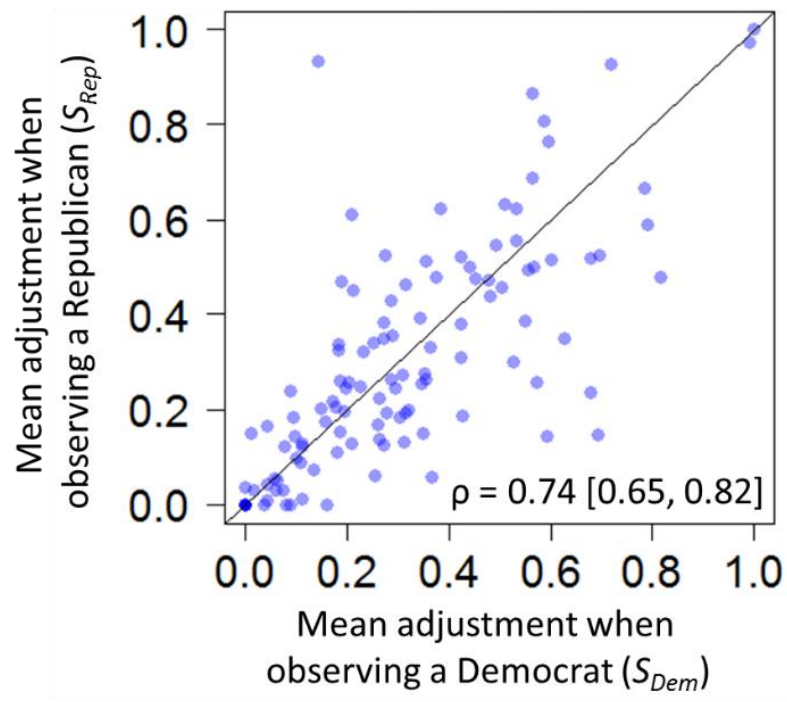

B

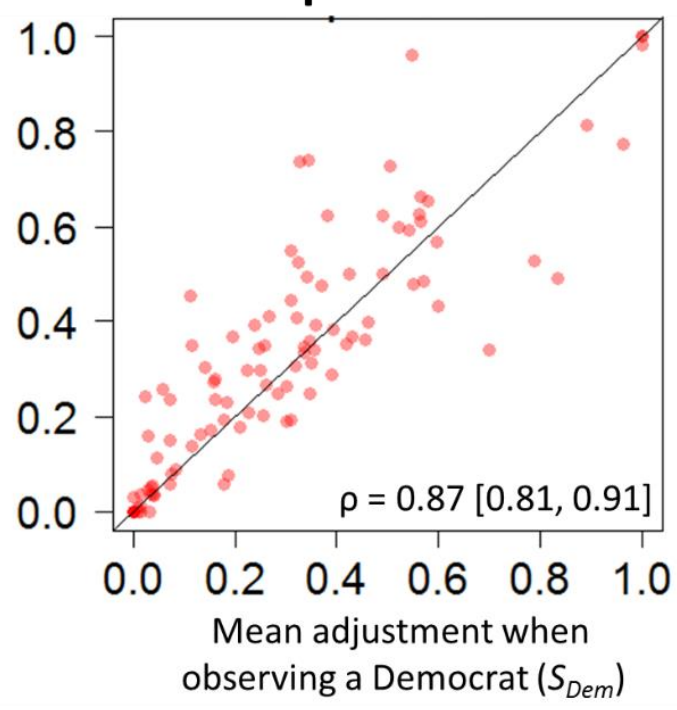

Fig. S6. Correlations of adjustments across experimental conditions. Each dot shows a participant's mean adjustment when social information was provided by a Republican as a function of the mean adjustment when it was provided by a Democrat. The two panels show data from participants who identified as Democrats (panel A) and Republicans (panel B). We observe strong consistency in social information use across both conditions. Numbers on the bottom right of each panel indicate Pearson's correlations, with $95 \%$ confidence intervals in brackets. 


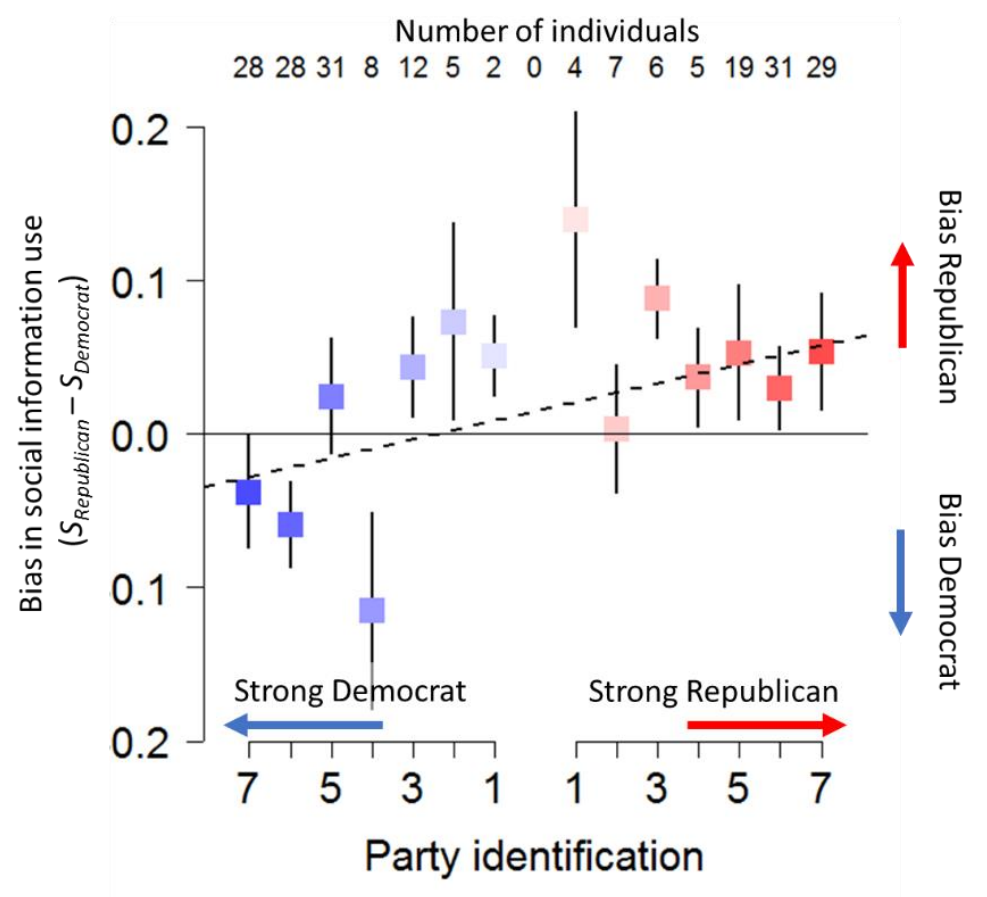

Fig. S7. The degree of partisanship predicts bias in social information use. The plot shows mean bias in social information use ( $B$; see main text) as a function of party identification $(P I)$. The variable $P I$ ranges from strong Democrat (coded as -7 ) to strong Republican (coded as +7 ). Squares indicate mean biases for each cohort, and bars indicate standard errors; numbers up top show the number of participants for each cohort. When $P I$ is considered on this continuum from -7 to 7 , a linear model fitted to $B$ reveals a significant positive association between $P I$ and $B$ (linear model, $b=0.004 ; P=0.027$ ). However, this result should be interpreted with caution, due to its small size, the potential qualitative transition in PI between -1 (weak affiliation with Democrats) and +1 (weak affiliation with Republicans), and the relatively large proportion of observations for extreme values of $\mathrm{PI}$. 


\begin{tabular}{|c|c|c|c|c|}
\hline Party & Mean age (s.d.) & Males & Females & Total \\
\hline Democrats & $37.2(10.5)$ & 64 & 52 & 116 \\
\hline Republicans & $41.6(12.0)$ & 54 & 50 & 105 \\
\hline
\end{tabular}

Table S1. Demographic details of the experimental sample. 


\begin{tabular}{|c|c|c|c|}
\hline & Model 1 & Model 2 & Model 3 \\
\hline Ingroup source (IS) & $\begin{array}{c}0.030(0.010,0.051) \\
p=0.004\end{array}$ & $\begin{array}{c}0.030(0.010,0.051) \\
p=0.005\end{array}$ & $\begin{array}{c}0.031(0.010,0.051) \\
p=0.004\end{array}$ \\
\hline Favored direction (FD) & $\begin{array}{c}0.025(0.004,0.045) \\
p=0.022\end{array}$ & $\begin{array}{c}0.026(0.004,0.046) \\
p=0.019\end{array}$ & $\begin{array}{c}0.025(0.004,0.046) \\
p=0.022\end{array}$ \\
\hline IS x FD & $\begin{array}{c}-0.015(-0.044,0.014) \\
p=0.322\end{array}$ & $\begin{array}{c}-0.016(-0.049,0.013) \\
p=0.284\end{array}$ & $\begin{array}{c}-0.015(-0.044,-0.014) \\
p=0.320\end{array}$ \\
\hline Uncertainty & & $\begin{array}{c}0.050(0.031,0.061) \\
p<0.001\end{array}$ & \\
\hline Same winner & & $\begin{array}{c}0.016(-0.001,0.033) \\
p=0.066\end{array}$ & \\
\hline Expertise & & $\begin{array}{c}0.002(-0.012,0 .-17) \\
p=0.743\end{array}$ & \\
\hline Population size & & $\begin{array}{c}-0.007(-0.015,0.000) \\
p=0.060\end{array}$ & \\
\hline Age (y) & & & $\begin{array}{c}-0.001(-0.003,0.002) \\
p=0.625\end{array}$ \\
\hline Gender male & & & $\begin{array}{c}-0.020(-0.083,0.043) \\
p=0.536\end{array}$ \\
\hline Gender other & & & $\begin{array}{c}-0.263(-0.729,0.203) \\
p=0.269\end{array}$ \\
\hline Intercept & $\begin{array}{c}0.289(0.255,0.322) \\
p<0.001\end{array}$ & $\begin{array}{c}0.252(0.152,0.351) \\
p<0.001\end{array}$ & $\begin{array}{c}0.320(0.205,0.450) \\
p<0.001\end{array}$ \\
\hline Observations & 2,757 & 2,644 & 2,757 \\
\hline Log likelihood & 329.27 & 307.53 & 321.51 \\
\hline AIC & -646.53 & -595.06 & -625.03 \\
\hline BIC & -611.00 & -536.27 & -571.73 \\
\hline
\end{tabular}

Table S2. Preregistered analyses. Linear mixed models fitted to adjustments (s; see Fig. 1 for formal definition) in individual rounds, with 'participant' as random intercept. Model 1 is the simplest model, in which only the central predictors enter the regression. Model 2 is the main model, and is graphically depicted in Fig. 2B (but the estimates of Ingroup source (IS), Favored direction (FD) and their interaction are very similar across models). Note that Fig. 2B includes the predictor 'Uncertainty', allowing to gauge 
effect sizes of IS and FD relative to a binary measure of confidence (1=low, $0=$ high), a factor shaping social information use which is well-documented in the literature (e.g., Morgan et al 2012). Models 2 and 3 include controls: 'Same winner' indicates for each state whether social information did or did not indicate the same majority outcome for that state as the participant's own first prediction (respectively coded as 1 and 0 ; cases where either first predictions or social information indicated a vote distribution of 50-50 were omitted). 'Expertise' reflects participants' self-rated knowledge of US politics on a 1-10 scale. 'Population size' indicates state population size (millions of inhabitants). Note that we deviated from the preregistration by not including a main effect of 'Degree of party identification' in any of the models. This variable is only informative in interaction with IS and/or FD (which we did not pre-register; see Fig. S7 for an alternative analysis). 


\begin{tabular}{|l|c|c|}
\hline & Democrats & Republicans \\
\hline Ingroup source (IS) & $\begin{array}{c}0.011(-0.018,0.040) \\
p=0.446\end{array}$ & $\begin{array}{c}0.053(0.024,0.083) \\
p<0.001\end{array}$ \\
\hline Favored direction (FD) & $\begin{array}{c}0.000(-0.030,0.030) \\
p=0.989\end{array}$ & $\begin{array}{c}0.057(0.026,0.087) \\
p<0.001\end{array}$ \\
\hline IS x FD & $\begin{array}{c}0.006(-0.035,0.048) \\
p=0.763\end{array}$ & $\begin{array}{c}-0.043(-0.085,-0.001) \\
p=0.048\end{array}$ \\
\hline Uncertainty & $\begin{array}{c}0.044(0.018,0.071) \\
p=0.002\end{array}$ & $\begin{array}{c}0.056(0.029,0.083) \\
p<0.001\end{array}$ \\
\hline Same winner & $\begin{array}{c}0.014(-0.009,0.037) \\
p=0.241\end{array}$ & $\begin{array}{c}0.016(-0.009,0.040) \\
p=0.215\end{array}$ \\
\hline Expertise & $\begin{array}{c}-0.138(-0.325,0.049) \\
p=0.148\end{array}$ & $\begin{array}{c}0.211(-0.022,0.445) \\
p=0.077\end{array}$ \\
\hline Population size & $p=0.393$ & $\begin{array}{c}-0.001(-0.002,-0.000) \\
p=0.043\end{array}$ \\
\hline Intercept & $\begin{array}{c}-0.001(-0.002,0.002) \\
p<0.001\end{array}$ & $\begin{array}{c}0.121(-0.041,0.284) \\
p=0.144\end{array}$ \\
\hline Observations & $0.357(0.235,0.480)$ & 1,239 \\
\hline
\end{tabular}

Table S3. Partisan biases in integrating social information. Numbers show estimates from two linear mixed models fitted to adjustments in individual rounds, respectively considering data from participants identifying as Democrats and Republicans. The first four predictors are shown in Fig. 3B of the main text. Predictors are the same as the main preregistered model for all data (Table S2, Model 2). The models reveal that the observed effects of ingroup bias in integration of social information (Fig. 2B) are driven by Republicans. 


\section{Experimental materials}

Below we show on-screen instructions as displayed to participants. In the week prior to the US 2020 General Elections, we posted a Human Intelligence Task (HIT) on Amazon Mechanical Turk (MTurk). This HIT was accessible to participants from the US who had an approval rating of $95 \%$. It indicated that the task would take about 20 minutes to complete, minimum and maximum earnings and a link to the experimental pages. At the end of the experiment, participants received a unique random code to collect their performance-specific payment on MTurk.

Where appropriate, we add explanations in boxes with a grey background. 


\section{Welcome!}

We are conducting an online study on the US Presidential Elections of 2020.

In this task, you will be presented with a selection of States and you will have to predict what voters will do.

Informed consent:

- By checking this box you give us informed consent that we can use your answers in anonymized form for research purposes only.

Next

Instructions: $10 \%$

The study is split into two general sections:

1. Prediction Task

2. Exit Questions 
In the Prediction Task you will be asked to predict how many out of 100 voters will vote for the Democratic or the Republican party for a selection of 20 States.

In total, you will play 20 rounds. Each round is divided in two parts, part A and part B.

Please note: In this study we only consider the Republican and Democratic voters. Other parties will get a share of votes, but for simplicity's sake here we only consider the two main Parties.

Instructions:

In part A of each round, you will use a slider to indicate how many out of 100 voters you think will vote for the Republican or the Democratic party.

Below, we see a participant entering their prediction for Colorado.

They predict $\mathbf{6 0}$ out of 100 voters to choose Democratic, and the remaining $\mathbf{4 0}$ to choose Republican.

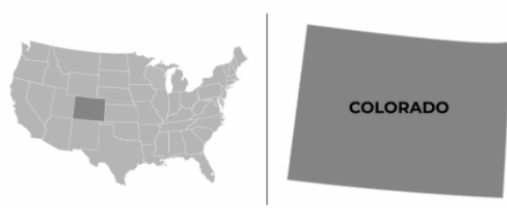

Make your part A prediction: out of 100 voters from Colorado, how many will vote Democrat or Republican?

Note: To avoid any anchoring effects, the slider for indicating the prediction was not initialized at any value (both in these instructions and in the main task).

In the next few screens, participants could familiarize themselves with using the slider. 


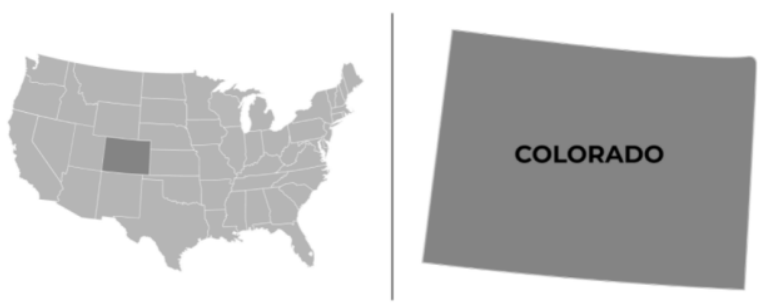

Make your part A prediction: out of 100 voters from Colorado, how many will vote Democrat or Republican?

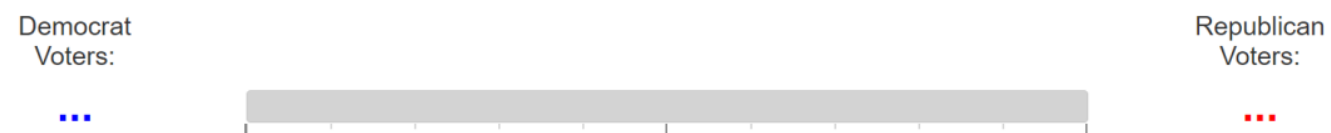

Submit

Instructions: $40 \%$

Dragging the slider handle to the right indicates that you expect more Democrat voters.

Democrat

Voters:

$\cdots$

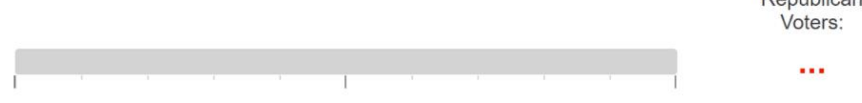

If you expect more Republican voters, drag the slider handle to the left.

Please note: you first have to click on the slider to activate it.

Press Next to try this out. 
Instructions:

$50 \%$

\section{A bit about money!}

At the end of the Task we will randomly select one of your predictions to determine your bonus prize!

If this prediction is exactly right, you earn $\$ 1.00$.

For each number (vote) you are off, we subtract $\$ 0.05$.

So, the closer you are to the actual outcome, the more money you will earn.

Press Next for an example.

Next

Instructions:

$60 \%$

Imagine you predicted that in Colorado 67 out of 100 voters will vote Democrat, and 33 Republican.

If the election outcome is that $60 \%$ of voters in that state actually voted Democrat, it means you were $67-60=7$ votes off.

Therefore, we would subtract $7 \times \$ 0.05=\$ 0.35$ from your bonus, and you would earn $\$ 0.65$. Please note, your bonus cannot become negative. If you are more than 20 votes off, and that round gets randomly selected, your bonus will be $\$ 0.00$.

In short, the closer you are to the actual outcome, the more money you will earn.

Any bonus you earn will be paid on top of the $\$ 1.70$. You'll only of course get this once the election results are out.

Back Next


After indicating your prediction, you will be asked to express your confidence in it. You have 2 options: Low confidence and High confidence.

Your confidence influences the chance that a part A prediction will be the one that is chosen for bonus payment.

If you express Low confidence in your prediction, it will have a normal chance to be the one that is selected for the bonus payment.

If you express High confidence in your prediction, it will be twice more likely to be the one that is selected for the bonus payment.

We will select only one of your part A decisions for payment. Which one that is depends on your confidence ratings.

The next few screenshots are from a mock participant familiarizing themselves with the decision screen.

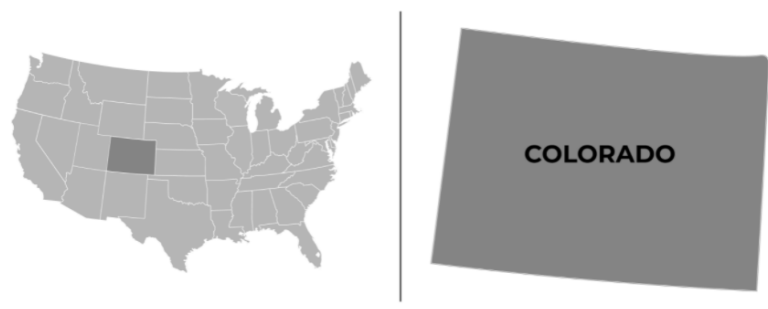

Make your part A prediction: out of 100 voters from Colorado, how many will vote Democrat or Republican?

\section{Democrat}

Voters:
Republican

Voters: 

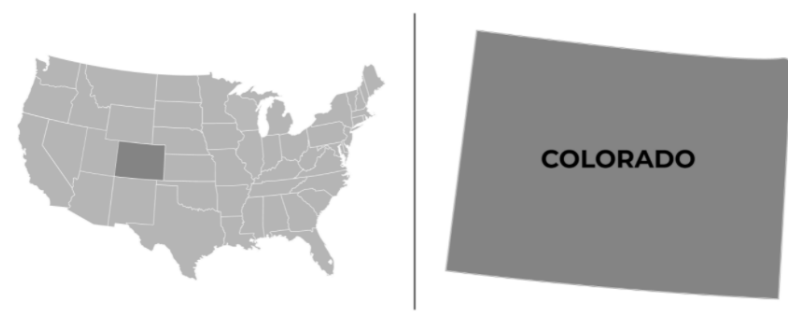

Make your part A prediction: out of 100 voters from Colorado, how many will vote Democrat or Republican?

Democrat

Voters:

53
Republican Voters:

47

Submit

By clicking the slider, a slider thumb appeared. Participants dragged this thumb left or right to indicate their estimate. Running numbers appear on the left and the right to show the values (Democratic Voters vs Republican Voters). 

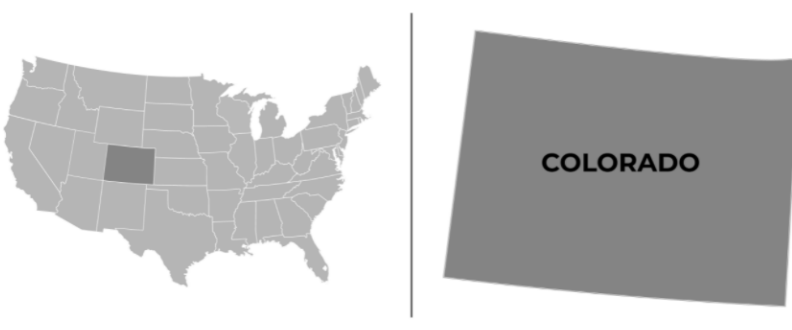

Make your part A prediction: out of 100 voters from Colorado, how many will vote Democrat or Republican?

Democrat

Voters:

53
Republican

Voters:

Please rate your confidence in this prediction.

Low High

Instructions: $\quad 80 \%$

Once you express your confidence in your prediction, part A of the round is over, and part B begins.

In part B you will observe the part A prediction of another MTurker.

Over 100 MTurkers participated in a previous session in which they completed this task.

In each round, you can observe the part A prediction of one of these previous MTurkers.

Their prediction was made for the same state you will see in that round.

They could also earn a higher bonus if their estimate was more accurate. 
Next to their prediction, you will also see their political affiliation: whether they identify more with the Republican or the Democratic party.

Please note: each round you will see the prediction of a different MTurker.

ALL the prediction you will see, are made by MTurkers who expressed High confidence.

Instructions: $100 \%$

In part B you have to make a second prediction.

You can make the same prediction as in part A, or adjust it as you wish.

Once you have made your part B prediction, the round is over and a new round begins.

IMPORTANT: You can win an additional bonus of $\$ 1.00$ for your part B prediction.

This bonus will be calculated in the same way as your part A bonus:

one of your predictions will be randomly selected, and the closer the prediction is to the real outcome, the higher your bonus will be.

In total, you can win up to $\$ 2.00$ in bonus $(\$ 1.00$ for part $A$, and $\$ 1.00$ for part $B$ ).

Click Next for a full practice round.

Back

Next

The next few screenshots are from a mock participant completing a full practice round. 


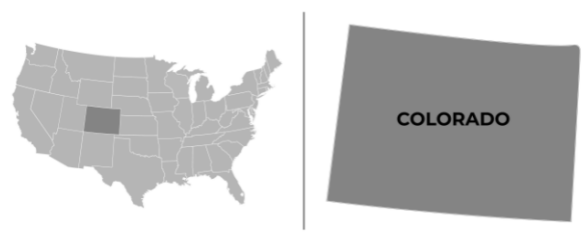

Make your part A prediction: out of 100 voters from Colorado, how many will vote Democrat or Republican?

Democrat

Voters:

47

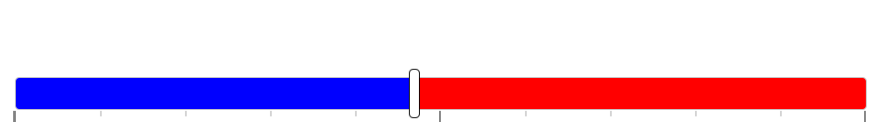

$\sqrt{1}$ Submit
Republican Voters:

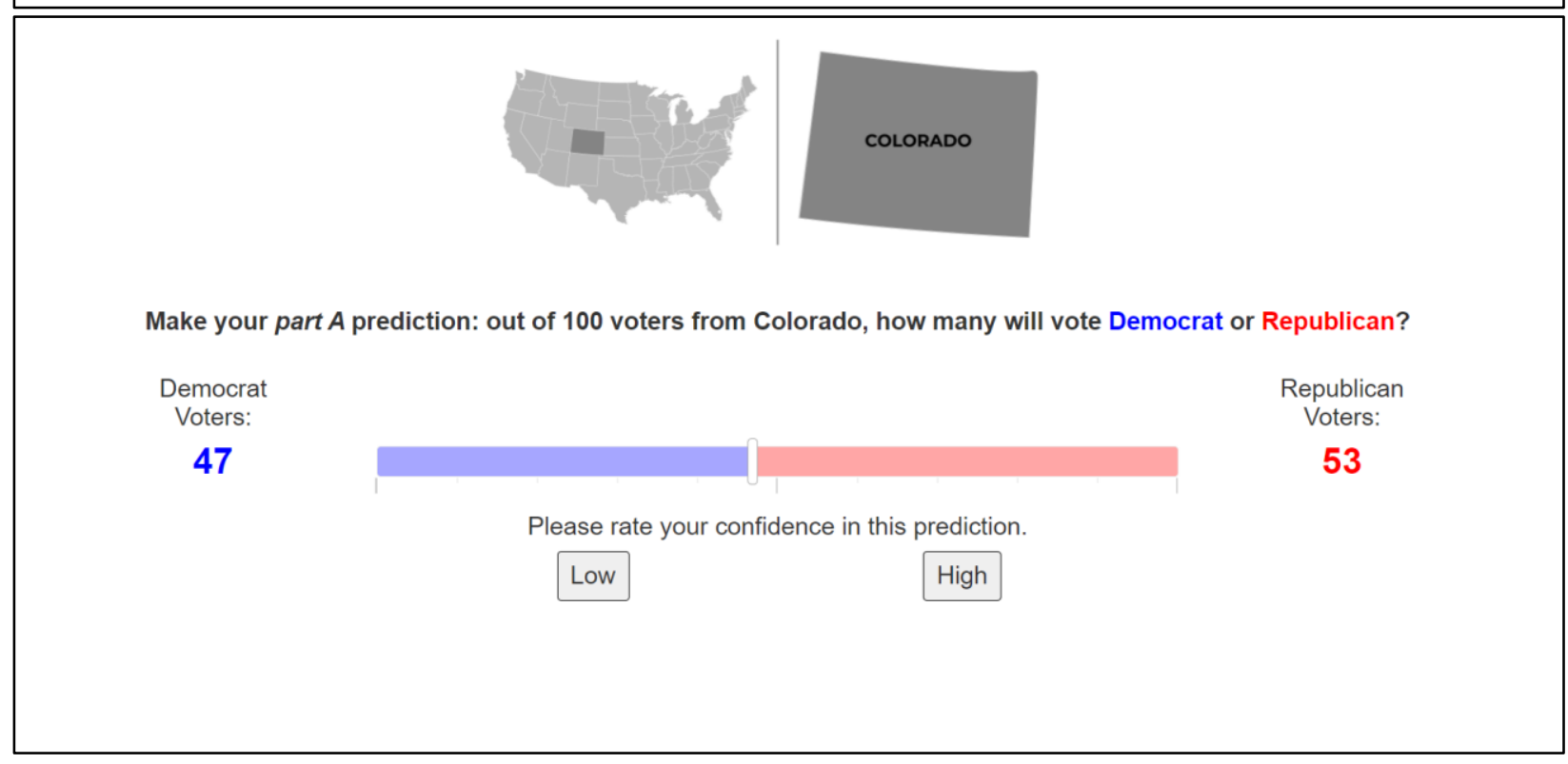


The slider on top starts at your part A prediction.

OK
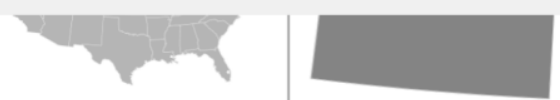

Make your part B prediction: out of 100 voters from Colorado, how many will vote Democrat or Republican?

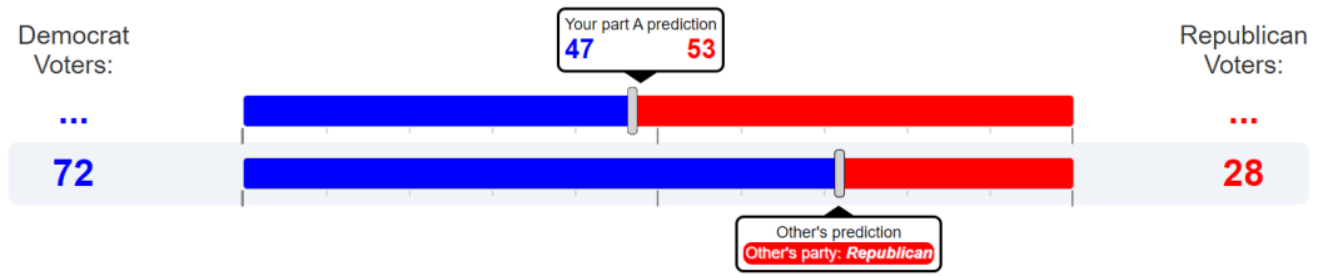

The slider on the bottom shows the prediction of another MTurker.

The box below their slider shows their political affiliation.

The horizontal grey bars are 'overlays' on the page. Participants had to click 'OK' make them disappear and proceed with the practice round.

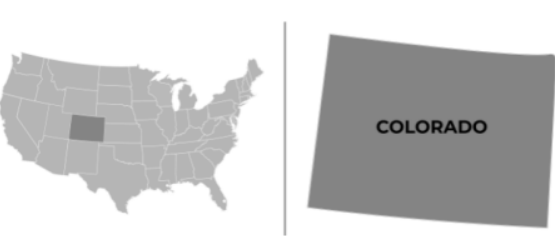

Make your part B prediction: out of 100 voters from Colorado, how many will vote Democrat or Republican?

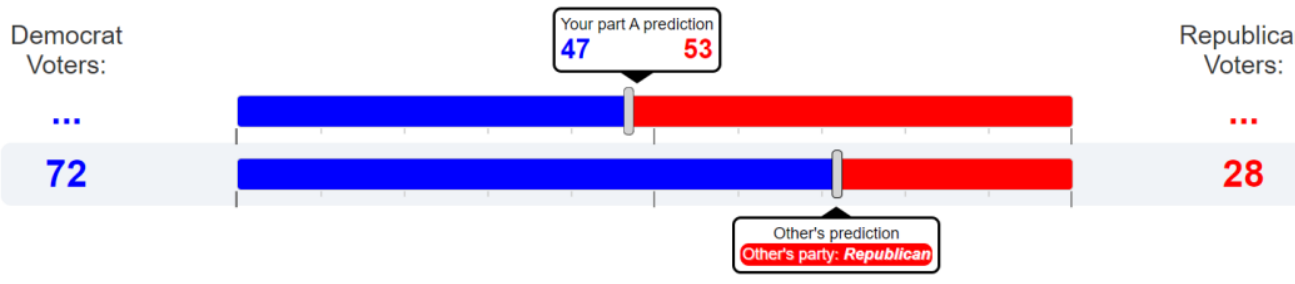

Participants made their second estimate by dragging the top slider. Running numbers appear on the left and the right to show the values (Democratic Voters vs Republican Voters). 


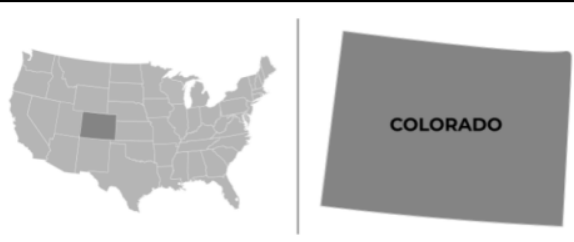

Make your part B prediction: out of 100 voters from Colorado, how many will vote Democrat or Republican?

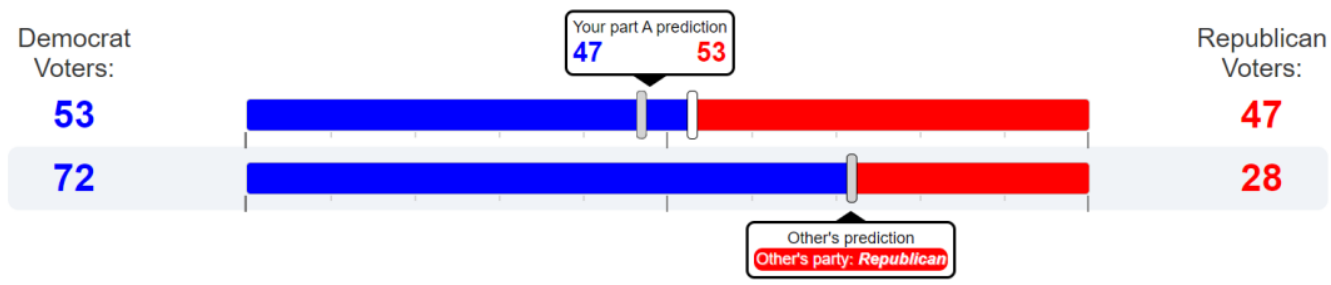

Submit

To check if you've understood the instructions, please answer the questions below.

In this task, you have to predict:

Who will win the elections How many out of 100 voters will vote for which Party

The closer your prediction is to the real outcome, the more bonus you can earn.

Correct Incorrect

At the end of this HIT we will select one of your Part A predictions for payment. Which one will be selected depends on your confidence: If you express High confidence in the part A prediction, that prediction will be twice more likely to be the one that is selected for the bonus payment.

Correct Incorrect

In part B of a round, you can observe the prediction of another MTurker who completed this task before you. You can then choose to revise your prediction.

Correct Incorrect

Next to their prediction, you will also see their political affiliation.

Correct Incorrect

Participants had to answer all control questions correctly to proceed to the task. Two entry questions about party affiliation were asked before the prediction task started. 


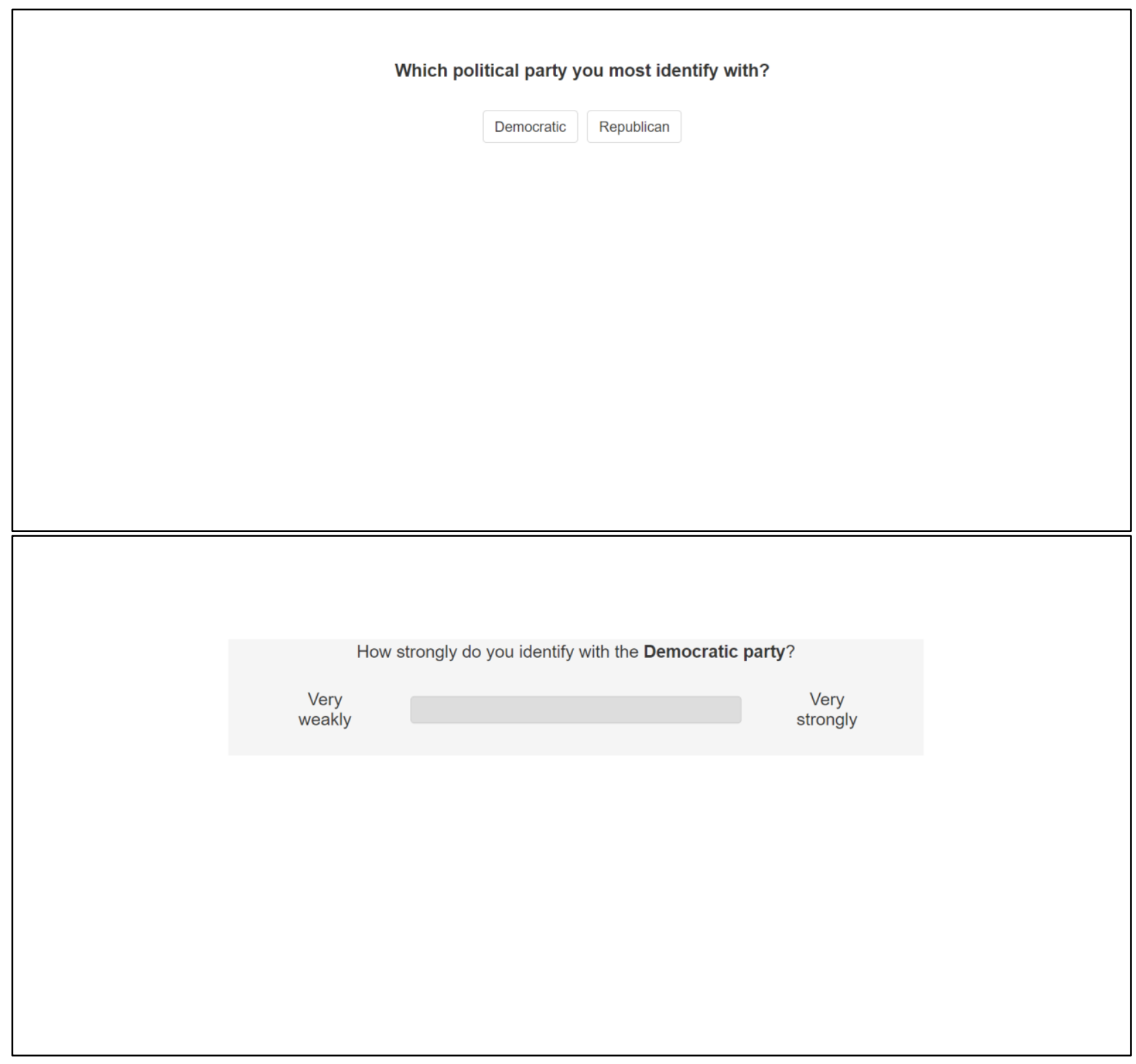

Page 25 of 32 


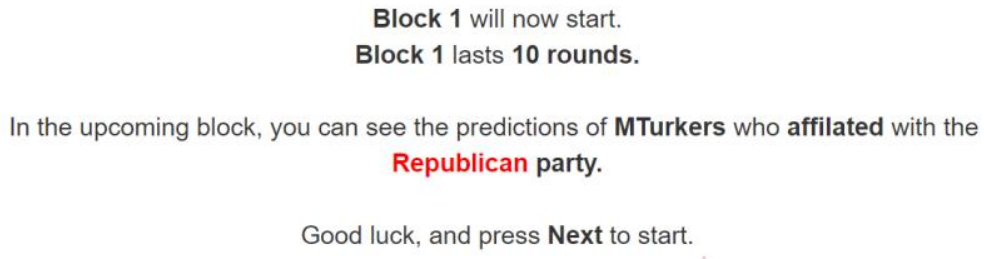

As described in the Methods, each block consisted of 10 rounds. After these 10 rounds, the end of the block was announced, and participants saw a screen exactly like this (with updated Block number and the other party affiliation of the MTurkers whose predictions could be observed). The blocks were counterbalanced in order.

To avoid unnecessary repetition, we show only one full round below. 


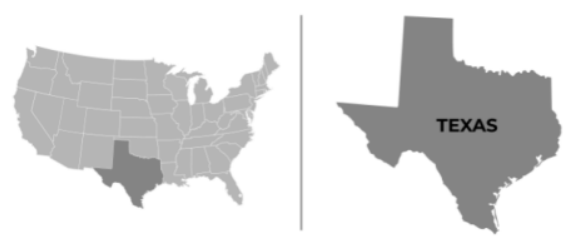

Make your part $A$ prediction: out of 100 voters from Texas, how many will vote Democrat or Republican?

Democrat

Voters:

$\cdots$
Republican

Voters:

$\cdots$

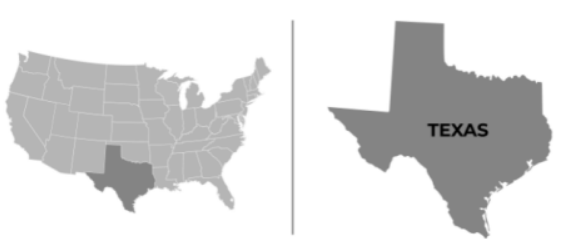

Make your part $A$ prediction: out of 100 voters from Texas, how many will vote Democrat or Republican?

Democrat

Voters:

46

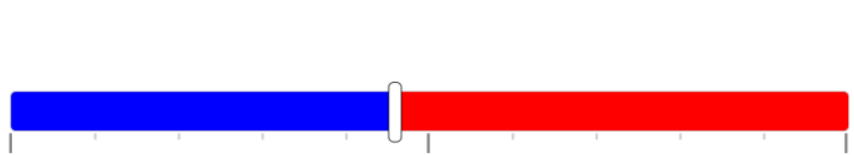

Republican Voters:

54 


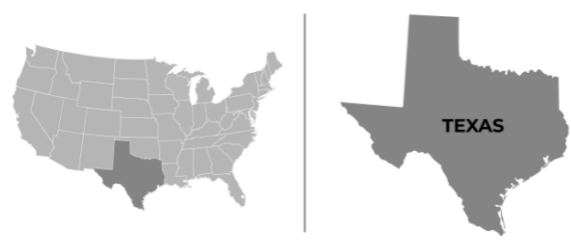

Make your part A prediction: out of 100 voters from Texas, how many will vote Democrat or Republican?

Democrat

Voters:

46
Republican

Voters:

Please rate your confidence in this prediction.

Low

High

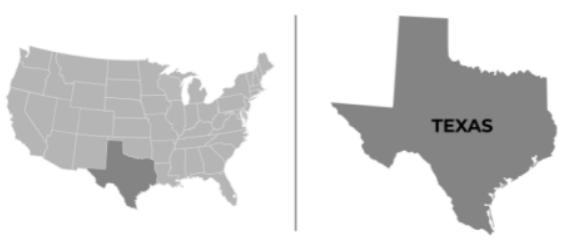

Make your part B prediction: out of 100 voters from Texas, how many will vote Democrat or Republican?

Democrat

Voters:

41

27
Your first prediction

46

54

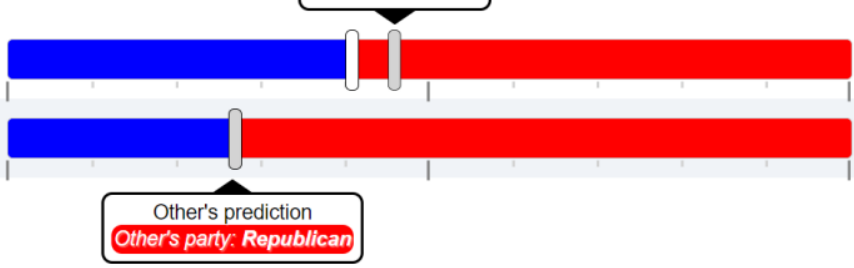

Republican Voters:

59

73

Submit 


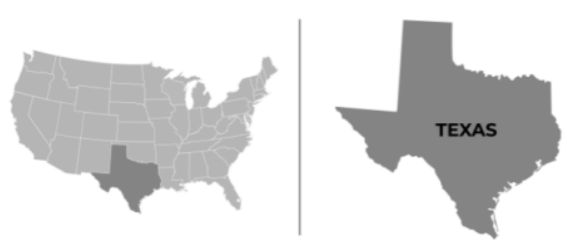

Make your part B prediction: out of 100 voters from Texas, how many will vote Democrat or Republican?

Democrat

Voters:

$\cdots$

27
Your first prediction

46

54

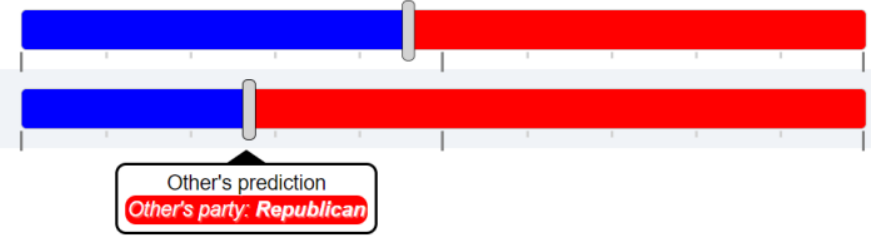

Submit
Republican

Voters:

$\cdots$

73

After the two blocks of 10 rounds each were completed, participants were asked to fill out an exit questionnaire. In our analyses we only include measures that we preregistered. 


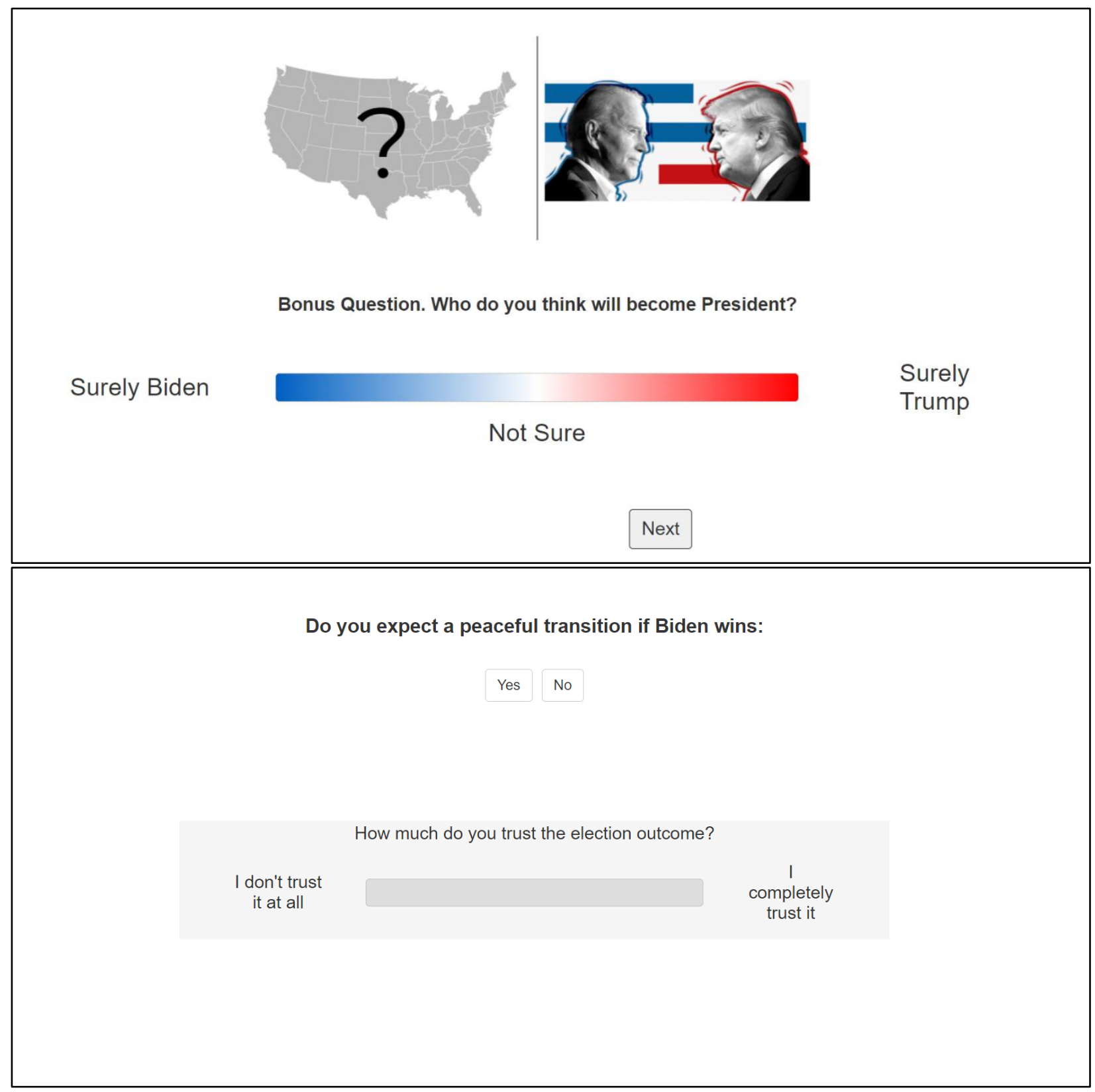


Where do you mostly get your news from?

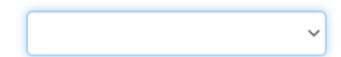

Apart from the above, do you have another major News source?

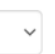

If a News source is not listed above, please state all sources here

Think of this ladder as representing where people from your country stand in terms of their knowledge of politics.

Click on the human icon on the rung where you think you should stand, relative to other people from your country.

The most knowledgeable people (who, for example, closely follow the news and daily read newspapers) are at the top, the least knowledgeable people are at the bottom.

Where would you place yourself on this ladder?

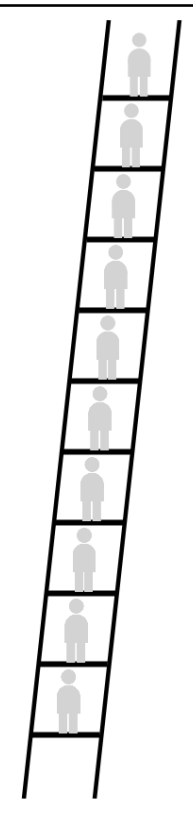

This 'ladder' measures the variable 'expertise' (cf. Table S2,3). 


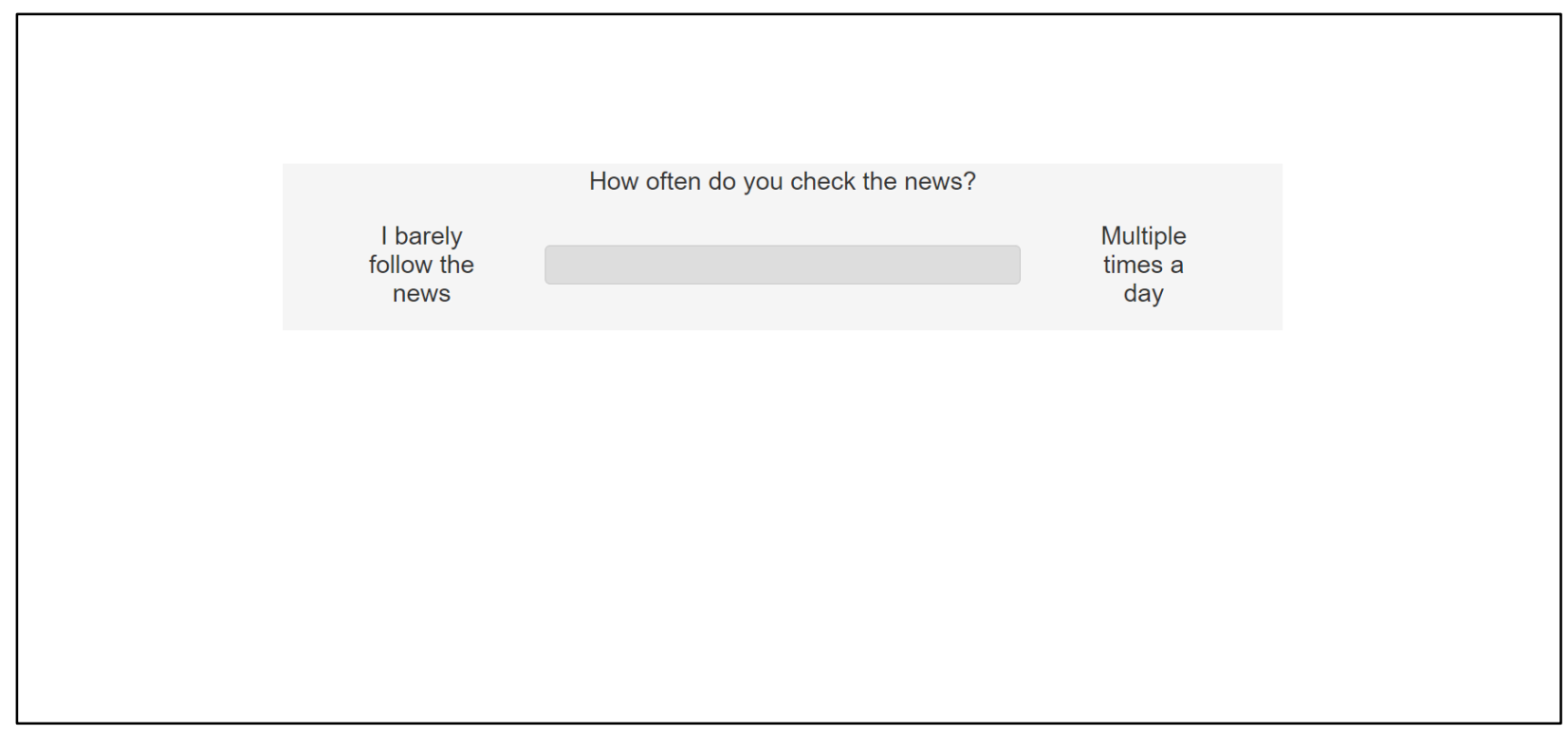

Page 32 of 32 